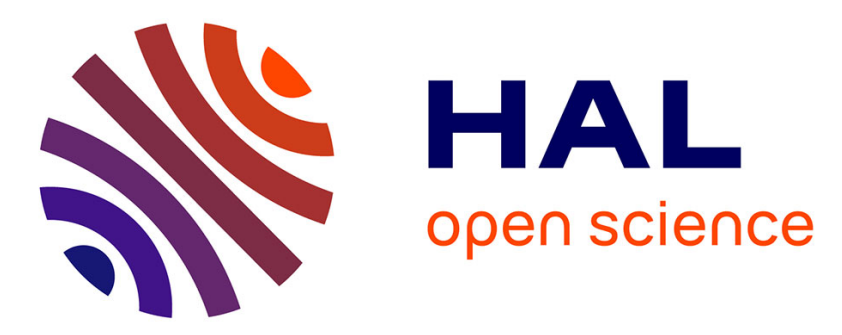

\title{
Van Hove singularities in intersubband transitions in multiquantum well photodetectors
}

J Le Rouzo, I Ribet-Mohamed, N Guerineau, R Haidar, M Tauvy, E Rosencher, Sl Chuang

\section{- To cite this version:}

J Le Rouzo, I Ribet-Mohamed, N Guerineau, R Haidar, M Tauvy, et al.. Van Hove singularities in intersubband transitions in multiquantum well photodetectors. Applied Physics Letters, 2006, 88 (9), 10.1063/1.2181647 . hal-01810811

\section{HAL Id: hal-01810811 \\ https://hal.science/hal-01810811}

Submitted on 6 Dec 2021

HAL is a multi-disciplinary open access archive for the deposit and dissemination of scientific research documents, whether they are published or not. The documents may come from teaching and research institutions in France or abroad, or from public or private research centers.
L'archive ouverte pluridisciplinaire HAL, est destinée au dépôt et à la diffusion de documents scientifiques de niveau recherche, publiés ou non, émanant des établissements d'enseignement et de recherche français ou étrangers, des laboratoires publics ou privés. 
See discussions, stats, and author profiles for this publication at: https://www.researchgate.net/publication/234948749

\section{Van Hove singularities in intersubband transitions in multiquantum well photodetectors}

Article in Applied Physics Letters · March 2006

DOI: $10.1063 / 1.2181647$

CITATIONS

10

7 authors, including:

Judikaël Le Rouzo

Aix-Marseille Université

92 PUBLICATIONS 423 CITATIONS

SEE PROFILE

Nicolas Guerineau

The French Aerospace Lab ONERA

148 PUBLICATIONS 1,539 CITATIONS

SEE PROFILE

Some of the authors of this publication are also working on these related projects:

Project multi-lateral shearing interferometry View project

Project $\quad$ FUN-PV Team View project
READS

152

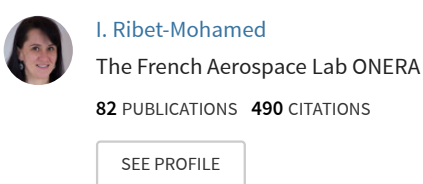




\title{
Van Hove singularities in intersubband transitions in multiquantum well photodetectors
}

\author{
Judikaël Le Rouzo, ${ }^{\text {a) }}$ I. Ribet-Mohamed, N. Guérineau, R. Haïdar, \\ M. Tauvy, and E. Rosencher ${ }^{\text {b) }}$ \\ Office National d'Etudes et de Recherches Aérospatiales, 91761 Palaiseau, France \\ S. L. Chuang \\ Department of Electrical and Computer Engineering, University of Illinois, 1406 West Green Street, \\ Urbana, Illinois 61801
}

(Received 25 July 2005; accepted 23 January 2006; published online 3 March 2006)

\begin{abstract}
Photocurrent spectra of quantum well infrared photodetector (QWIP) devices have been studied over more than three orders of magnitude, revealing features which have been largely overlooked before. Electric field assisted tunneling and, more surprisingly, Van Hove singularities at the miniband edges are shown to play an important role in the low and high energy parts of the QWIP photocurrent spectra, respectively. The photoresponse of QWIPs away from their peak responsivity is found to be non-negligible ( $>1 \%$ in the 3-5 $\mu \mathrm{m}$ for a 8-12 $\mu \mathrm{m}$ detector), which has to be taken into consideration when optimizing multispectral devices. (C) 2006 American Institute of Physics.
\end{abstract}

[DOI: $10.1063 / 1.2181647]$

Multiquantum well infrared photodetectors (QWIPs) are now a mature technology for large infrared focal plane arrays. $^{1-4}$ Among the main advantages of this technology, one finds the possibility of multispectral detection, thanks to stacked structures with different growth parameters. ${ }^{5}$ For this purpose, it is very important to determine and understand the photoresponse of QWIPs with great accuracy away from their peak responsivity. Indeed, this will allow one to evaluate the possible crosstalk between the signals originating from different detected infrared bands (3-5 and 8-12 $\mu \mathrm{m}$ for instance). However, mainly because of experimental difficulties, very little effort has been devoted to measure and modeling these off-band responsivities with sufficient dynamical range. In this letter, we describe an experiment (referred as Log spectra) which allows these measurements. Thanks to the large dynamical range, these Log spectra show that the off-band spectral responsivity of QWIPs is largely influenced by quantum effects such as electric field assisted tunneling for the low energy side and, rather unexpectedly, by Van Hove singularities due to the quantum well periodicity for the high energy side.

The QWIP detectors have been elaborated by Thales Research and Technology laboratory. They consist in $40 \mathrm{GaAs}$ quantum wells, $5 \mathrm{~nm}$ thick with $35 \mathrm{~nm} \mathrm{Al}_{0.26} \mathrm{Ga}_{0.74} \mathrm{As}$ barriers. The structure was designed so that the first excited state is quasibound in the well, for a responsivity peak at $8.5 \mu \mathrm{m}$. The following results would nevertheless apply to bound-toextended transitions. Bound-to-bound transitions are more complex because of additional phenomena, such as sequential tunneling. ${ }^{6}$ In order to determine the intrinsic spectral responsivity of the QWIPs, $100 \mu \mathrm{m} \times 100 \mu \mathrm{m}$ mesas are defined by reactive ion etching without any grating coupling. The QWIP is mounted in a JANIS continuous flow helium cryostat placed outside a Fourier-transform infrared spectrometer (FTIR). This commercial FTIR (Bruker, Equinox IFS55) produces either a reference spectrum (thanks to an

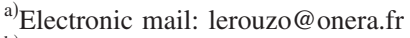

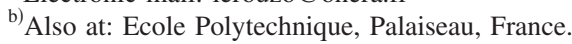

internal reference detector with a known spectral response), or an external collimated beam of uniform intensity which is used to illuminate the detector. The angle of incidence on the sample may be adjusted from normal incidence to $\pm 50^{\circ}$. The time-dependent signal delivered by the detector in response to the time-varying interferogram is amplified (Keithley 428) and sent to a series of passband filters to get rid of noise. It is then sent to the spectrometer for Fourier transformation and normalization by the reference spectrum. The transmission of the cryostat germanium window is taken into account in our measurements.

Figure 1 shows the Log spectra of QWIPs for different applied biases. For comparison purpose, the spectra are normalized to their peak responsivities. This figure illustrates the good dynamical range of our measurements since three orders of magnitude are explored thanks to our low noise experimental setup. The main features of the figures are the following: First, the experiment confirms the non-negligible

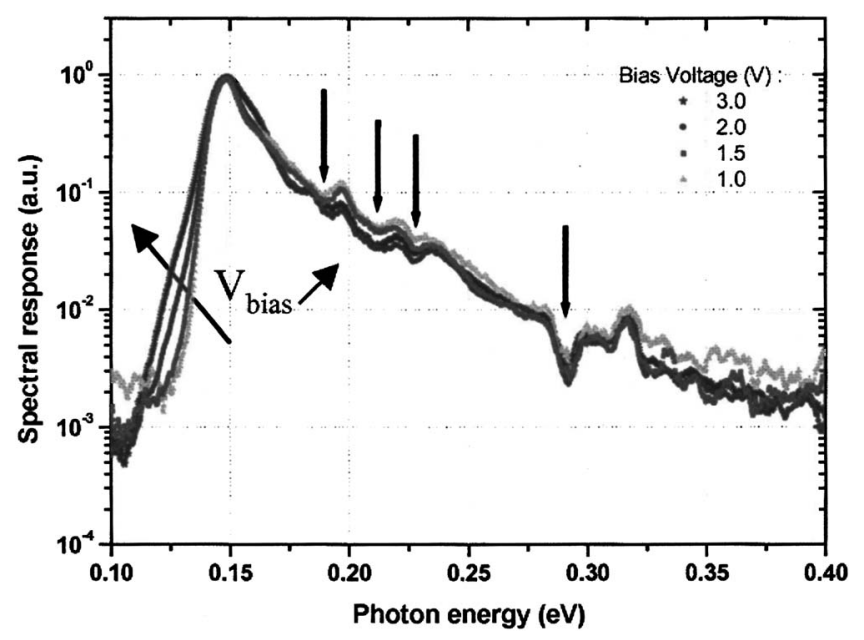

FIG. 1. Experimental Log photocurrent spectra of typical QWIP samples for different applied electric fields $(1,1.5,2,3 \mathrm{~V})$. Note the influence of the electric field in the low energy part and the sharp features indicated by arrows in the high energy part. 


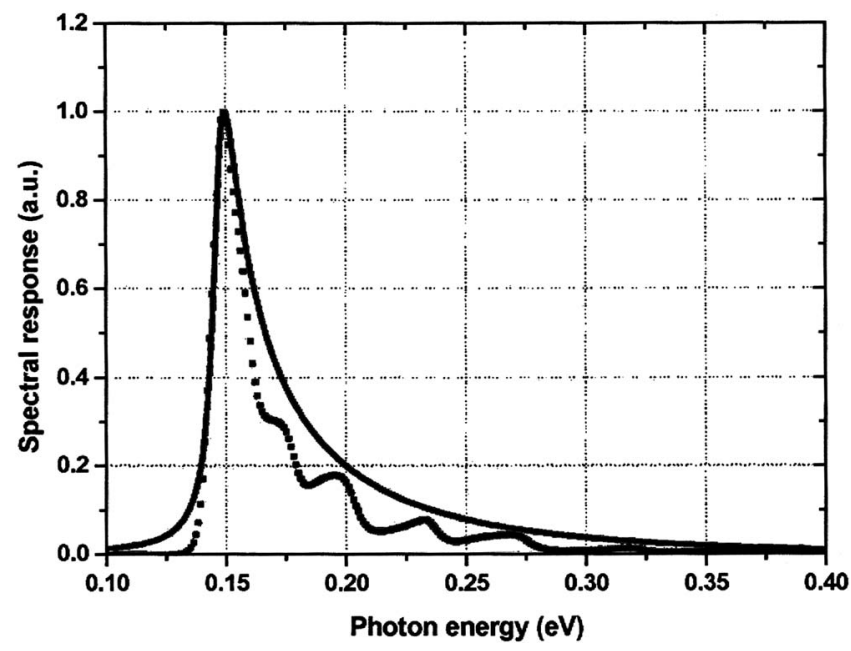

FIG. 2. Comparison between the absorption spectrum of the QWIP calculated in a single quantum well (continuous line) and in a periodic QWIP structure (dotted line). The QWIP parameters are: $5 \mathrm{~nm}$ thick GaAs QW with $35 \mathrm{~nm} \mathrm{Al}_{0.26} \mathrm{Ga}_{0.74} \mathrm{As}$ barriers and broadening coefficient $\Gamma=5 \mathrm{meV}$.

off-band response of the QWIP in the 3-5 $\mu \mathrm{m}$ window compared to its resonance value (still $1 \%$ in the $3-5 \mu \mathrm{m}$ range). This value, however, is likely to be attenuated using coupling gratings. Second, the low energy side of the spectral response is strongly affected by the applied electric field while the high energy side shows little dependence. Finally, rather sharp features are observed in the high energy part of the spectra as indicated by arrows in Fig. 1. These features show very little dependence on the applied bias. Apart from the well documented $\sin ^{2} \theta$ dependence of the QWIP responsivity, ${ }^{8}$ no influence of the angle of incidence is observed on these spectral features.

Concerning the low energy part of the Log spectra, the displacement of the photocurrent cutoff points to electric field assisted tunnelling (or Fowler-Nordheim effect). This mechanism has already been mentioned in the QWIP literature concerning the dark current, ${ }^{9}$ the escape probability from quantum well, ${ }^{10,11}$ or the injection mechanism at the injecting contact. ${ }^{12}$ However, this feature has not been clearly identified in photocurrent spectra. As far as the high energy part of the Log spectra is concerned, the spectral features are reminiscent of Van Hove singularities. This will be confirmed by the following theoretical model.

We have developed a simple model based on two main ingredients: electric field assisted tunneling and Van Hove singularities. Since we are interested in the spectral aspects of the responsivity, we have not included the already well known transport mechanisms such as electric field redistribution in the QWIP structure or carrier injection at the blocking barrier. ${ }^{13,14}$ Indeed, these theoretical ingredients are primordial for describing the quantitative photoresponse of the QWIP but bring little information on the spectral shape. The photocurrent spectral shape is thus taken to be proportional to the oscillator strength between the bound state and the exited states with peak responsivity normalized to 1 .

Since the features are not affected by the electric field, we made the assumption that they are due to the quantum well periodicity and not to any complex quantum effect calling for a complete solution of the Schrödinger equation of this complex structure under an applied electric field. This quantum interference between the periodic quantum wells is Downloaded 07 Mar 2006 to 144.204.16.1. Redistribution subject

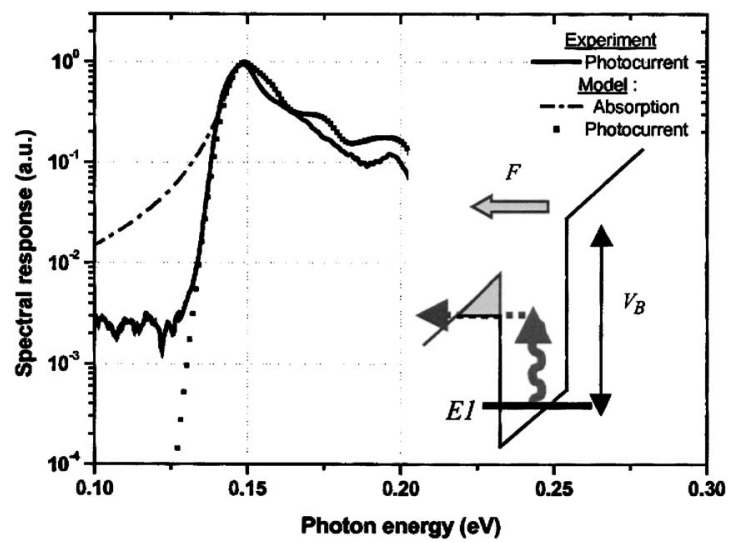

FIG. 3. Comparison between an experimental QWIP Log spectrum (continuous line), the theoretical absorption (dashed line), and the theoretical photocurrent spectra (dotted line) taking into account the electric field assisted tunneling effect (see inset).

known to be the basis of superlattice photodetectors-where quantum well are coupled by tunneling ${ }^{15}$-but is generally neglected in QWIP modeling. We have thus chosen a simple Kronig-Penney model to describe the states in the quantum wells. ${ }^{16}$

Let $|n, k\rangle=u_{n, k}(z) e^{i k z}$ be the Bloch quantum state of wave vector $k$ in the $n$th band. The function $u_{n, k}(z)$ displays the periodicity of the QWIP structure, i.e., $u_{n, k}(z+L)=u_{n, k}(z)$ with $L=b+d$, where $d$ and $b$ are the quantum well and barrier widths, respectively. Since a single level is bound in the quantum wells, the $n=1$ state is the bound state while the $n>1$ states are the free ones. The exact expressions of the function $u_{n, k}(z)$ and of the energy of the $|n, k\rangle$ states [i.e., $\left.E_{n}(k)\right]$ can be found in text books, such as Expressions (4.7.20) in Ref. 16. The momentum matrix element between the bound state $|1, k\rangle$ and the excited state $\left|n, k^{\prime}\right\rangle$ is given by

$$
p_{1, n}\left(k, k^{\prime}\right)=\left|\left\langle 1, k\left|\frac{A \hat{p}_{z}}{m}\right| n, k^{\prime}\right\rangle\right|,
$$

where $A$ is the infrared light vector potential, $m$ the electron mass and $\hat{p}_{z}=(\hbar / i)(d / d z)$ is the momentum operator which has to be used in periodic structures. ${ }^{8,15}$ A straightforward calculation shows that

$$
\begin{aligned}
p_{1 n}\left(k, k^{\prime}\right) & =p_{1 n}(k) \\
& =\frac{A}{m} \hbar\left|\int_{\text {cell }} u_{1, k}(z) * \frac{d}{d z} u_{n, k}(z) d z\right| \delta\left(k, k^{\prime}\right),
\end{aligned}
$$

where $\delta$ is the Kronecker symbol and $\hbar$ is the Planck constant. The integral is evaluated over a single quantum well period (cell). The Fermi Golden Rule provides the probability $P(h \nu)$ of the electron excitation by the incident photon flux of energy $h \nu, P(h \nu)=(2 \pi / \hbar) p_{1 n}(k)^{2} \rho\left(E_{1}+h \nu\right)$, where $\rho(E)$ is the density of states in the periodic structure. The absorption spectrum is then the convolution of the excitation probability $P(h \nu)$ with a Lorentzian line shape of broadening parameter $\Gamma$. In order to illustrate the importance of the QW periodicity, we compare in Fig. 2 this absorption spectrum calculated by our Kronig-Penney approach with that obtained using expression (10) in Ref. 17 which neglects this periodicity. The used QWIP parameters are $5 \mathrm{~nm}$ thick GaAs QW with $35 \mathrm{~nm} \mathrm{Al} \mathrm{Al}_{0.26} \mathrm{Ga}_{0.74} \mathrm{As}$ barriers and $\Gamma=5 \mathrm{meV}$. The AlP license or copyright, see http://apl.aip.org/apl/copyright.jsp 
figure clearly shows the Van Hove singularities at the miniband edges.

The low energy part of the photocurrent spectrum is obtained by requiring that electrons photoexcited below the barrier energy $V_{B}$ may tunnel through the electric field triangular barrier using the Fowler-Nordheim transmission coefficient [see expression (4) in Ref. 9 for instance]. Figure 3 shows the comparison in the low energy part between the theoretical absorption, the photocurrent, and an experimental Log spectrum, respectively. The agreement is convincing and the influence of the Fowler-Nordheim effect is clearly illustrated. Photons with energy below the ionization threshold $V_{B}-E_{1}$ (see inset of Fig. 3) are absorbed but do not yield a photocurrent in the absence of an electric field: This leads to a cutoff in the photocurrent spectrum compared to the absorption one. This cutoff is gradually moving toward lower energy with the increasing electric field (Fowler-Nordheim effect) which explains the low energy features of Fig. 1.

Figure 4 shows the comparison between an experimental Log spectrum and our theoretical model, taking into account both Fowler-Nordheim and Van Hove singularities effects. The overall agreement is rather good, particularly concerning the position of the Van Hove singularities. However, two points raise questions: First, there is still an important quantitative discrepancy between our theoretical model and the

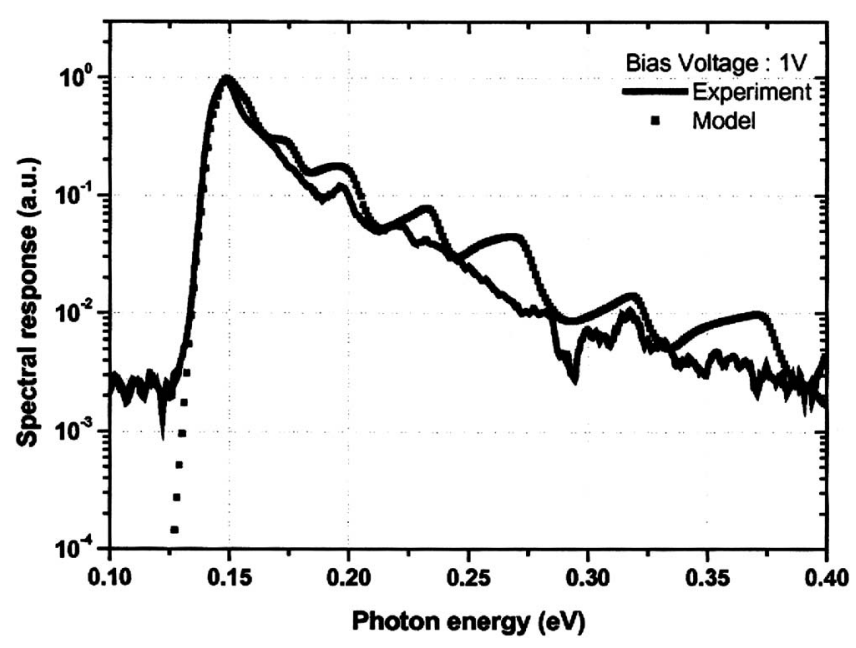

FIG. 4. Comparison between an experimental Log spectrum (continuous line) and our theoretical model taking into account the electric field assisted tunneling effect and the Van Hove singularities at the miniband edges (dotted line). experimental Log spectra on the high energy side. This discrepancy is most certainly due to different contributions such as (i) band nonparabolicity and (ii) energy dependent mobility of the free carriers. The second point is the rather low value of the determined broadening coefficient $\Gamma$. Indeed, the rather sharp features observed in the Log spectra are compatible with a value of $5 \mathrm{meV}$ but not with the usual value of $10 \mathrm{meV}$.

In conclusion, photocurrent spectra of QWIP devices have been studied over more than 3 decades. These Log spectra reveal features which have been largely overlooked before. Electric field assisted tunneling and Van Hove singularities - somewhat reminiscent of quasibound states observed by Capasso et al. in electron Fabry-Pérot type structures ${ }^{18}$ - are shown to play an important role in the low and high energy parts of the QWIP photocurrent, respectively. The off-band photoresponse is found to be non negligible ( $>1 \%$ in the $3-5 \mu \mathrm{m}$ for a $8-12 \mu \mathrm{m}$ detector) but smaller than expected by the theory.

The authors are indebted to the TRT Thales team $(\mathrm{Ph}$. Bois, E. Costard) for providing the QWIP samples.

${ }^{1}$ B. F. Levine, J. Appl. Phys. 74, R1 (1993).

${ }^{2}$ Intersubband Transitions in Quantum Wells, edited by H. C. Liu, B. Levine, and J. Andersson (Plenum, London, 1994), pp. 97-110.

${ }^{3}$ J. L. Pan and C. G. Fonstad, Jr., Materials Science and Engineering Reports 28, 65 (2000).

${ }^{4}$ K. K. Choi, Physics of Quantum Well Infrared Photodetectors (World Scientific, Singapore, 1997).

${ }^{5}$ S. D. Gunapala, S. V. Bandara, J. K. Liu, M. Jhabvala, and K. K. Choi, Infrared Phys. Technol. 44, 411 (2003).

${ }^{6}$ K. K. Choi, B. F. Levine, R. J. Malik, J. Walker, and C. G. Bethea, Phys. Rev. B 35, 4172 (1987).

${ }^{7}$ I. Ribet-Mohamed, N. Guérineau, S. Suffis-Carretero, M. Tauvy, A. De Rossi, E. Costard, and P. Bois, J. Appl. Phys. 97, 023106 (2005).

${ }^{8}$ E. Rosencher and B. Vinter, Optoelectronics (Cambridge University Press, Cambridge, 2002).

${ }^{9}$ S. R. Andrews and B. A. Miller, Appl. Phys. Lett. 70, 993 (1991).

${ }^{10}$ E. Martinet, E. Rosencher, F. Chevoir, J. Nagle, and Ph. Bois, Phys. Rev. B 44, 3157 (1991).

${ }^{11}$ B. F. Levine, Intersubband Transitions in Quantum Wells, edited by E. Rosencher, B. Vinter, and B. F. Levine (Plenum, London, 1992).

${ }^{12}$ E. Rosencher, F. Luc, Ph. Bois, and S. Delaitre, Appl. Phys. Lett. 61, 468 (1992).

${ }^{13}$ M. Ershov, C. Hamaguchi, and V. Ryzhii, Jpn. J. Appl. Phys., Part 1 35, 1395 (1996)

${ }^{14}$ L. Thibaudeau, P. Bois, and J. Y. Duboz, J. Appl. Phys. 79, 446 (1996).

${ }^{15}$ M. Helm, Semicond. Sci. Technol. 10, 557 (1995).

${ }^{16} \mathrm{~S}$. L. Chuang, Physics of Optoelectronic Devices (Wiley Interscience, New York, 1995).

${ }^{17}$ H. C. Liu, J. Appl. Phys. 73, 1 (1993).

${ }^{18}$ F. Capasso, C. Sirtori, J. Faist, D. L. Sivco, S.-N. G. Chu, and A. Y. Cho, Nature (London) 358, 565 (1992). 